\title{
La communication touristique, une triple invention
}

\author{
Philippe Viallon
}

\section{OpenEdition}

\section{Journals}

Édition électronique

URL : http://journals.openedition.org/tourisme/171

DOI : $10.4000 /$ tourisme 171

ISSN : 2492-7503

\section{Éditeur}

Éditions touristiques européennes

\section{Édition imprimée}

Date de publication : 1 juin 2013

Pagination : 2-11

ISSN : 2109-5671

\section{Référence électronique}

Philippe Viallon, « La communication touristique, une triple invention », Mondes du Tourisme [En ligne] 7 | 2013, mis en ligne le 30 septembre 2015, consulté le 21 décembre 2020. URL : http:// journals.openedition.org/tourisme/171 ; DOI : https://doi.org/10.4000/tourisme.171

\section{(ब) $\Theta \Theta$}

Mondes du tourisme est mis à disposition selon les termes de la licence Creative Commons Attribution - Pas d'Utilisation Commerciale - Pas de Modification 4.0 International. 


\title{
La communication touristique,
} une triple invention

\author{
PHILIPPE VIALLON_ [viallon@unistraff] \\ Professeur en sciences de l'information et de la \\ communication à l'Université de Strasbourg
}

Résumé. Bien connue des professionnels, qui la conçoivent comme un aspect du marketing, la communication touristique est un concept encore peu développé par les chercheurs. Pourtant, depuis plus de cinquante ans, les sciences de l'information et de la communication produisent des travaux sur les médias, les organisations, les archives ou la circulation de l'information (sous sa forme numérique, tout particulièrement), qui peuvent être rapportés au champ du tourisme avec intérêt. Ce texte envisage quelques pistes pour ce faire.

Abstract. Tourism communication is a well-known concept among professionals who think of it as being related to marketing, but it is rarely used by researchers. Over the last fifty years a lot of research has been produced in the fields of communication and information science concerning the media, organizations, the archives, and the circulation of information, in particular digital information. This research can be applied to the domain of tourism in a productive manner. This text explores some of the possibilities opened up by this approach. 


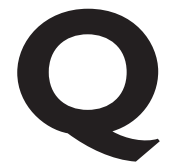

u'est-ce que la communication touristique ? Les mots ne semblent pas poser problème. L'expression est encore relativement peu employée (Jaworksi et Pritchard, 2005 ; Luger, 2000 ; Boyer et Viallon, 1994) dans le monde de la recherche qui, pourtant, parle de communication politique, de communication des organisations ou de marketing touristique et de politique touristique... Dans le monde professionnel, l'expression est courante et comprise comme une partie du marketing touristique, plus précisément comme une de ses phases opérationnelles, lorsque les stratégies décidées trouvent leur matérialisation sur des supports visant des publics restreints avec des objectifs précis.

Pour la recherche, les apparences trompent et la question est plus complexe qu'il n'y paraît. La rencontre de ce que les sociologues (Bourdieu, 1976) appellent des "champs", la communication et le tourisme, appelle, en effet, une série de réflexions : Quelles conséquences la rencontre de ces deux champs amène-t-elle d'un point de vue épistémologique ? Dans quelle forme de communication le tourisme se situe-t-il ? Comment les professionnels conçoivent-ils cette communication, notamment avec les technologies de l'information et de la communication (Tic) ? Enfin, comment les touristes, autrefois apparemment passifs, utilisent-ils ces mêmes Tic pour s'inventer une autonomie ?
Pour tenter de cadrer ces questions, cette chronique va se développer en deux temps. Dans une première partie sera soulignée une identité épistémologique frappante entre les deux champs : il s'agit de deux inventions, celle de la communication et celle du tourisme. Dans une deuxième partie sera envisagée l'association des deux concepts qui amène une troisième invention, celle de la communication touristique. Ce nouveau (?) concept a des conséquences si importantes pour l'étude du tourisme qu'on ne peut que reconnaître la nécessité d'inclure la communication touristique à toute réflexion de haut niveau sur cette activité humaine et à toute formation au tourisme.

\section{DEUX INVENTIONS}

\section{L'invention \\ de la communication}

Les hommes ont communiqué de tout temps, mais la communication comme science n'existe pas depuis les origines de l'homme. Il faut donc différencier, d'une part, la formalisation de techniques de communication, de l'Antiquité grecque et latine au $\mathrm{XX}^{\mathrm{e}}$ siècle, et, d'autre part, l'analyse systématique et holistique du processus de communication, qui a pris le relais à partir de la première moitié du $\mathrm{XX}^{\mathrm{e}}$ siècle avec la constitution d'une science.

Les chercheurs (Mattelart, 1994; Breton et Proulx 1993) sont d'accord pour voir dans la rhétorique aris- totélicienne la première formalisation de l'argumentation et des techniques de conviction. Les concepts d'ethos, de logos et de pathos (Aristote), par exemple, mettent en relief les ressorts du discours de conviction et sont encore aujourd'hui pertinents pour analyser le discours publicitaire touristique : l'ethos correspond à l'image que l'émetteur donne de lui-même à travers son discours et renvoie à sa crédibilité et la confiance qu'il doit éveiller auprès du récepteur ; le logos couvre l'argumentation mise en place et les stratégies afférentes ; le pathos renvoie aux sentiments mobilisés et à tout l'affect de la communication. Barthes (1957) les a d'ailleurs appliqués avec justesse aux trois éléments clefs de la communication : l'émetteur, qui doit avoir une éthique pour être crédible, le message, qui doit comporter de bons arguments, et le récepteur, qui se laisse toucher ou non par le message. Aristote souligne aussi les mauvais usages possibles de la rhétorique, telle que la pratiquent par exemple les sophistes. Cicéron, dans sa volonté d'expliquer comment un avocat doit parler pour convaincre, a aussi insisté sur la nécessité d'organiser son discours, de connaître son auditoire... Les pères de l'église, et notamment Thomas d'Aquin, ont développé pour leur part la scolastique, que l'on peut considérer comme une forme religieuse de la rhétorique.

La philosophie des Lumières et la Révolution française ont eu à cœur de diffuser dans le peuple la 
connaissance, le savoir et les idées, dont les doctrines économiques des physiocrates. Ils ont compris que les idées circulaient mieux lorsque ceux qui les portaient pouvaient emprunter des routes en bon état et ont donc incité au développement d'un réseau routier, montrant la nature fondamentalement duale de la communication, entre matérialité et immatérialité. Les réflexions de Saint-Simon (1819) sur les relations entre êtres humains qui dépendent de la capacité du réseau à établir le lien appartiennent aussi champ de la communication. Cependant, comme le soulignent Breton et Proulx (1993), "le discours qui fait de la communication une valeur centrale, à laquelle il est nécessaire de recourir systématiquement pour résoudre toutes sortes de problèmes sociaux et économiques, est, quant à lui, d'apparition historique récente".

Ce discours est daté dans la première moitié du XX' siècle. L'École de Chicago, tout d'abord, a fortement contribué à expliquer des phénomènes de masse, avec l'étude des villes et de leur sociologie, celle des migrations, de la déviance (criminologie), l'étude du travail, ainsi que de la culture et de l'art. La cybernétique, ensuite, a permis une modélisation de l'échange, par l'étude de l'information et des principes d'interaction (Wiener, 1952). Si l'on y ajoute d'autres modèles linéaires, comme ceux de Shannon (1948) ou de Jakobson (1963), toute la dimension mécaniste est mise à jour. Lasswell (1927) et ses cinq questions ("Qui (dit) ? quoi ? (à) qui ? (par) quel moyen ? (avec) quel effet ?”), Lazarsfeld et Katz (1955) et leur two-steps-flow, selon lequel les médias n'influencent pas directement le public, mais atteignent des leaders d'opinion qui, eux, vont avoir un effet sur le public, approfondissent encore la dimension communicationnelle. D'autres études, notamment celles dites "systémiques", pour lesquelles l'interaction, la totalité (le tout est plus important que la somme de ses parties), l'organisation et la complexité sont au centre des réflexions, y contribuent également.

Pour l'école de Palo Alto, “on ne peut pas ne pas communiquer" (Watzlawick, 1988). Dans les années 1960, ce sont les cultural studies puis les gender studies qui vont s'attacher à la compréhension des comportements des individus, d'une part, et le structuralisme puis le constructivisme qui vont apporter une nouvelle perspective philosophique à la discipline, d'autre part. Désormais, les sciences de l'information et de la communication (Sic) s'attachent surtout à comprendre les conséquences du développement des Tic dans la société et les usages qu'en font les individus.

En faisant une synthèse de toutes ces approches, on peut aujourd'hui avoir une vision très précise des phénomènes en jeu, que ce soit au niveau des personnes concernées, du mode diffusion, du coût, de l'espace couvert, des effets possibles. Il y a donc bien une historicité des sciences de l'information et de la communication. Mattelart a donc raison de parler d'une "invention de la communication" (1994). Dans les année 1970 et 1980, les chercheurs du domaine se sont beaucoup interrogés sur la nature des Sic (discipline, interdiscipline, transdiscipline...) ; ces interrogations quasi existentielles, que l'on retrouve dans l'histoire des sciences à chaque nouvelle naissance, ont permis de poser des bases solides articulant objets, paradigmes, théories et méthodes.

Les Sic, comme le souligne Dacheux (2009), ont "pour objet de comprendre non pas 'les' outils de communication, mais 'la' communication. Il s'agit donc, pour les Sic, de restituer toutes les dimensions (symboliques, politiques, économiques)".

Les paradigmes mis en place peuvent être soit communs aux sciences sociales, soit spécifiques aux Sic, tels la scientificité de la démarche, le positionnement de l'homme au centre de la réflexion, le caractère systémique des objets analysés ou la dimension constructiviste de la réalité, pour n'en citer que quelquesuns. Les théories ont été déjà évoquées dans le rappel historique : on peut y ajouter celles, plus récentes, de l'ethnographie des audiences (Livingstone, 1998), des uses and gratifications, les études féministes ou la théorie du réseau (Musso, 2003). Quant aux méthodes, elles sont largement communes avec les autres sciences humaines et sociales : analyses de discours, de contenus et d'images, approches 
quantitatives et qualitatives, entretiens, enquêtes et observation participante constituent l'essentiel des outils.

Les Sic ont donc mis en place une recherche active qui s'interroge sur les théories, sur la pensée, voire sur les idées, même les plus proches du quotidien des individus, une discipline qui s'enseigne dans les structures de formation, qui forme des spécialistes et fait avancer les connaissances lors de colloques et séminaires. Elles ont également une présence de plus en plus grande dans les discours scientifiques, sociaux et professionnels et pour finir une capacité à se conjuguer avec d'autres champs comme celui du tourisme.

Dans le champ du tourisme, les objets étudiés par les Sic peuvent être partagés avec d'autres disciplines - géographie (Stock, 2003 ; Violier 2007), science politique (Nahrath et al., 2012; Clivaz et Nahrath, 2010) ou sociologie (Cousin, 2003). Ils font aussi partie d'ensembles beaucoup plus vastes qui nécessitent une approche interdisciplinaire, comme le prouve numéro après numéro la revue Mondes du tourisme.

\section{L'invention du tourisme}

Les pratiques touristiques existent depuis longtemps, mais le tourisme n'a pas toujours existé. Les riches Romains pratiquaient l'otium, l'oisiveté, qui était une marque de leur statut social ; ils allaient de Rome à leur maison de campagne dans le Latium et, par- fois, visitaient le grand empire romain. La noblesse, classe dirigeante du Moyen Âge, instaura des règles très strictes qui empêchaient les nobles d'avoir une activité marchande, sous peine de déroger. Les deux seuls métiers autorisés étaient le soufflage du verre et le commerce par voie de mer. Ces deux exceptions visaient à combler un vide dans l'activité économique. Le temps ainsi libéré servait à la guerre, souvent à l'étranger, mais aussi aux déplacements nombreux : l'histoire de la Cour de France est remplie de longs voyages. Les voyages étaient aussi pour des motifs religieux, les pèlerinages. Mais c'est une pratique précise qui va créer le tourisme en tant que tel : au XVIII ${ }^{\mathrm{e}}$ siècle, les riches nobles anglais développent the tour (d'où le mot "tourisme"), voyage de formation que leurs fils accomplissent accompagnés de leur précepteur à travers le continent et qui, au terme d'un périple de un à deux ans, les mène en général à Rome. Boyer (1999) a montré comment ce "tour" était un moyen pour la noblesse de se distinguer, au sens "bourdieusien" du terme, de la bourgeoisie qui avait peu à peu conquis le pouvoir politique. Cette "invention du tourisme" (Boyer, 1996), est donc précisément datée et le phénomène va lentement se répandre à travers les couches sociales inférieures par imitation, par capillarité. L'appropriation par une nouvelle couche amène souvent une forme de rejet par la couche plus élevée, puisque la fonc- tion de distinction disparaît. En France, les couches populaires ont été atteintes en 1936 grâce aux fameux congés payés, même si cela n'a pas été le rush que l'on veut bien dire. Le tourisme se développe avec l'amélioration des conditions de vie : les Trente Glorieuses (Dumazedier, 1962) seront en France caractérisées par l'entrée définitive dans les mœurs du principe des vacances, par un développement du temps de vacances. Tissot (2007) parle, à propos du Club Med, "d'une utopie réalisée". D'un temps unique de vacances (le plus souvent en été), on est passé dans les années 1990 à un temps fractionné. L'abaissement du coût du transport aérien, du fait notamment de la libéralisation économique et du développement de nouveaux modèles économiques (cf. les charters, puis les vols low cost), a aussi largement contribué au changement des pratiques. Dans les pays émergeants, le désir de tourisme serait le deuxième souhait après celui de posséder une voiture, ce qui promet des flots importants de touristes chinois ou indiens ou brésiliens qui, à leur tour, devraient imiter les touristes occidentaux. Le tourisme est devenu aujourd'hui l'une des premières industries mondiales, un des rares domaines où une activité constante, voire croissante, semble assurée pour de nombreuses années malgré les aléas économiques ou politiques. Il fonctionne toujours selon le principe, celui d'une invention qui est copiée à l'infini. 


\section{L'INVENTION \\ DE LA COMMUNICATION TOURISTIQUE}

La communication et le tourisme sont bien des inventions, en ce sens que ce sont des créations ex nibilo à partir de l'imagination de quelques individus visionnaires, leaders d'opinion. Les nouveaux sports de glisse (venant souvent de Californie), les parcs à thèmes (type Disneyland), les visites virtuelles, la réalité augmentée sont autant de pratiques qui ont été ou sont en train d'être inventées. Ces inventions récentes associent étroitement tourisme et communication, que soit par les pratiques proposées, par les techniques utilisées, ou par le discours tenu à leurs propos.

La rencontre de ces deux inventions et de l'arrière-plan, théorique et scientifique, qui les accompagne ne se fait pas sans conséquence. On peut concevoir alors la communication touristique comme une nouvelle invention, qu'il s'agit de définir. Dans le cadre d'une approche interdisciplinaire revendiquée par de nombreux chercheurs en Sic et en tourisme, il est important que chaque discipline (sociologie, géographie, anthropologie, économie, histoire, marketing, droit...) explique ce qu'elle peut apporter à la construction du savoir sur le tourisme. C'est l'objectif de cette deuxième partie.

\section{La communication touristique comme approche alternative}

- Investir le tourisme par le biais des Sic permet de mettre à profit les résultats des recherches théoriques menées depuis plus de quarante ans, en France comme à l'étranger, dans de nombreux domaines : la constitution d'opinions, l'analyse de messages médiatiques, l'étude de la réception de ces messages, la place des nouvelles technologies dans la société, l'organisation des structures (entreprises, collectivités locales...). À partir du moment où le tourisme traite ces aspects, il ne sert à rien pour les chercheurs dans le tourisme de "réinventer la roue" ; il vaut mieux utiliser le savoir acquis et voir dans quelle mesure ces résultats peuvent s'appliquer au champ du tourisme. On soulignait en introduction le peu de tentatives qui ont été menées dans cette direction que ce soit en France, en Allemagne ou dans le monde anglo-saxon. Pourtant les enjeux humains et financiers sont importants.

La communication touristique permet une approche alternative, parmi d'autres, aux nombreux travaux de sociologie qui ne voient dans le tourisme que des aspects négatifs. De Veblen (1899) à Baudrillard (1991), en passant par Enzensberger (1965) et Barthes (1957), la liste est longue des chercheurs qui ne voyaient que l'aspect consumériste du tourisme et son impact négatif, sur l'environnement, sur les structures sociales des populations locales... Pour eux, le tourisme n'était qu'une soupape pour des individus aliénés par la société et un ballon d'oxygène de survie pour des populations locales exploi- tées par le capitalisme mondial. Ils allaient jusqu'à affirmer que "l'appareil du tourisme international interdit de façon organique toute relation réelle entre visiteur et visité" (Cazes, 1976). La sociologie plus récente, sans se détacher complètement de cette critique, aborde la question de manière plus complexe en développant la dimension anthropologique du tourisme : L'Idiot du voyage d'Urbain (|99|) est un bon exemple de cette évolution. La démarche critique correspondait à une période où l'on pensait qu'un refus en bloc de la pratique touristique pourrait la freiner. On sait aujourd'hui que ce n'est pas le cas et on a appris à voir dans le tourisme une dimension plus complexe qui permet d'envisager des pratiques qui satisfont les touristes comme les hôtes, qui limitent ses propres excès, voire qui sont plus respectueuses des hôtes et de leur l'environnement. Cette évolution a été en grande partie permise par ce que Violier (2007) appelle une "invention $d u$ regard".

- La communication touristique, comme d'autres disciplines, offre un autre point de vue, qui se distingue du marketing (Tocquer et Zins, 1987), sur l'activité touristique. Si certains économistes ont tendance à associer les deux, il existe des différences fondamentales comme, par exemple, la manière de décliner la place donnée au touriste, à l'homme. Penser le tourisme uniquement en termes de capacités hôtelières, de production de catalogues et de type d'acheminement 
renforce les critiques évoquées plus haut, exclut du processus touristique toute une partie importante des touristes potentiels, méconnaît les processus de fonctionnement de la prise de décision du départ. Cela ne veut pas dire que le marketing ne soit pas important pour l'activité touristique, mais son approche doit être complétée par une vision plus proche du fonctionnement réel des individus, une approche plus sociale. Il s'agit de contrebalancer les résultats d'une stratégie qui met le client et sa "demande" au centre des préoccupations par une vision plus complexe, antérieure à l'acte d'achat, qui se préoccupe de la représentation d'un lieu, d'un pays, d'une activité, d'un moyen de transport... En un mot, qui se préoccupe de l'image.

- Une autre différence essentielle consiste dans le fait que le marketing cherche plutôt à apporter une réponse immédiate à des problèmes essentiellement matériels. La communication touristique, sans s'éloigner des contingences économiques, aspire à une réflexion sur le long terme, prenant en compte la complexité des réalités, s'intéressant autant au touriste qu'à son hôte. Ce n'est pas le profit financier qui est son objectif principal, mais le profit humain et environnemental.

- La communication touristique est aussi un exemple particulièrement intéressant de communication interculturelle. La dimension interculturelle du tourisme reste un domaine largement inexploré. Les liens entre le développement du tourisme dans les pays occidentaux et l'importation massive de pratiques culinaires issues des pays visités restent encore à analyser ; la part de la connaissance directe de l'Autre, malgré tous les obstacles de la culture et de la langue, dans les représentations individuelles et sociales, est encore à étudier (par exemple, le tourisme des voyages linguistiques des jeunes et la vision de l'Europe qui en découle). La relation entre tourisme et immigration - touristes et immigrants sont des publics qui se retrouvent souvent dans les mêmes avions, les mêmes bateaux - a commencé à être étudiée (Viallon, 2008), mais il faudrait la mener plus loin. Le tourisme est également trop souvent restreint à celui qui se pratique entre pays de niveaux de vie différents ; en Europe, des pays comme la Suisse et l'Allemagne sont parmi les plus actifs, tant pour émettre que pour recevoir des touristes. Amirou (1995), Gravari-Barbas et Graburn (2012) ont bien inauguré la voie de l'analyse des représentations, des imaginaires du tourisme.

\section{Quelques pistes}

Comment orienter cette invention

de la communication touristique?

Une première possibilité est de construire un cadre théorique. Quels sont les développements récents des sciences de l'information et de la communication qui peuvent trouver une application dans le tourisme? En quoi des concepts de base comme le constructivisme ou la sys- témique peuvent-ils enrichir la problématique du tourisme ? Des chercheurs comme Bernard et Joule (2005) ont développé des concepts comme la "communication engageante" qui pourrait aussi intéresser le tourisme. Qu'est-ce que les nouvelles approches du tourisme en France, comme celles d'Urbain (1991, 1994) ou de l'Équipe Mit (2000, 2005, 20II), apportent au concept ? Faut-il aller plus loin et revendiquer une science du tourisme, une "tourismologie" comme le font certains (Hoerner, 2002) ou bien l'approche interdisciplinaire qui préside actuellement est-elle plus riche de développements que l'enfermement dans une discipline ? Quels sont les apports possibles des Sic et de leur dimension interdisciplinaire ? De nouveaux styles et manières d'interpréter le tourisme utilisent les langages du visuel (Rakic et Chambers, 2012) et du numérique (Morand et Mollard, 2008), ou des nouvelles approches de l'analyse quantitative (Baggio et Klobas, 20 I I). Avec quelles conséquences ? Enfin, des spécialistes problématisent de plus en plus l'application de méthodes particulières à des sphères sociales spécifiques où la communication n'est jamais absente, comme le développement économique (Brau, 2008), le marketing et l'hospitalité (Nykiel, 2007) ou le tourisme culturel (Richards et Munsters, 2010).

Une deuxième possibilité est d'étudier le tourisme comme forme de communication et de déterminer l'approche adaptée. Voyager est 
une forme de communication au monde et aux autres. Quels sont les éléments qui poussent les touristes à quitter leur lieu de résidence et pourquoi vont-ils vers telle ou telle destination ? Ces facteurs push et pull (Uysal et Jurowski, 1994) sont à la fois des explications à un phénomène migratoire qui concerne en 2013 un milliard de personnes (flux internationaux) (OMT, 20I2), mais aussi des signes qui renvoient à l'identité des individus et des groupes, à l'image qu'ils veulent donner d'eux-mêmes. En ce sens, les classifications habituelles de touristes, qui vont de l'explorateur au touriste de masse (Cohen, 1979), sont-elles satisfaisantes ? De leur côté, afin de répondre à des attentes qu'ils croient déceler chez leurs clients, les professionnels mettent de plus en plus en scène les espaces réels, les médiatisent ou en créent de virtuels (musée du Louvre, par exemple). Faut-il craindre une déréalisation du monde, une "disneylandisation" de la réalité (Brunel, 2006) ? Faut-il diluer le tourisme dans l'espace plus large des loisirs pour mieux le comprendre ou suffit-il simplement de le mettre dans cette perspective? Ne faut-il pas sortir de l'opposition réel-virtuel pour se pencher sur les pratiques et les offres, toutes bien réelles ? Comment alors penser la relation et l'articulation entre les univers matériels et électroniques, à travers notamment les offres de réalité augmentée ou tout simplement le passage de l'un à l'autre effectué en permanence par les touristes, de la collecte de l'information à la découverte des lieux ? Les études sur les réseaux sociaux numériques soulèvent la question de la méthodologie possible et permutable à l'intérieur des études sur le web 2.0 ou sur le web 3.0 (Andrade, 20 I I).

Il faut également comprendre le tourisme comme forme de communication, d'une part dans sa dimension ontologique, d'autre part dans sa dimension sociologique. Les émetteurs de discours touristiques sont multiples : les professionnels du secteur (tour-opérateurs, organismes de tourisme - du niveau national au niveau local -, les hôtels, restaurants, commerces... ), les professionnels d'autres secteurs (éditeurs pour les guides, journalistes, cinéastes, romanciers...), les particuliers qui passent du côté de l'offre (chambres d'hôtes, blogueurs, participants à un forum touristique...). Le support est aussi essentiel : le papier a longtemps était le principal support (sous forme de prospectus, brochure, affiche, catalogue...), tandis que l'audiovisuel restait à la marge. Désormais, le support numérique est en train de bouleverser cette hiérarchie. Du côté du récepteur, on oublie parfois que le tourisme ne se limite pas à la pratique en elle-même : il y a un "avant", fait de recherche d'informations, de comparaisons... et un "après" au cours duquel la constitution de la mémoire et le partage de l'expérience sont essentiels.

Un troisième aspect concerne la communication vue du côté des professionnels. Depuis 2009, plus de la moitié des transactions financières liées au tourisme en France se font sur internet (Stratégies, 2009). Comment aujourd'hui sont construits les sites web des professionnels du tourisme? Quels sont les choix faits dans la communication numérique par les structures publiques (États, régions, villes, offices de tourisme...) ou privées ? Comment se fait l'accueil des internautes de langue ou de culture étrangères ? Les nouvelles technologies modifient la donne : si les hôteliers sont moins soumis à la contrainte des guides papier traditionnels, ils le sont en revanche aux nouveaux guides (Tripadvisor), aux distributeurs en ligne (Booking, Expedia...) et aux règles du référencement sur le web. Comment apprécier ces changements ? Comment gérer les réseaux sociaux, dont l'importance semble ne pas s'arrêter de croître ? Comment émerger dans ce flot d'informations, comment gérer les techniques les plus récentes comme la géolocalisation, la mobilité, la réalité augmentée ? Les Tic ont bouleversé de nombreux domaines des sociétés contemporaines, notamment celui du tourisme. Peut-on entrer sans distance critique dans une idéologie techniciste selon laquelle le futur du tourisme sera meilleur grâce à la technique?

Un dernier point est représenté par la communication du côté des touristes. Grâce aux forums, aux blogs, aux sites d'avis de voyageurs (Tripadvisor) et autres réseaux sociaux (Facebook, Twitter...), le 


\section{CHRONIQUE SCIENTIFIQUE}

touriste potentiel n'est plus dépendant de la seule information officielle des professionnels de la branche ou des guides touristiques. Les études (Viallon et Serrano, 20 I 3 ; Viallon et Henneke-Lange, 20 I3) montrent que la crédibilité accordée aux autres internautes est souvent supérieure à celle des sites officiels. Pourquoi croire un internaute, que l'on ne connaît pas, plus qu'une structure qui a une réputation à conserver ? Comment penser cette transposition de la crédibilité, voire de la légitimité des experts, aux touristes amateurs ? Que peut-on en conclure sur la transformation des positions sociales et symboliques de la figure des amateurs, dont certains deviennent des amateurs experts ? Doit-on parler de démocratisation ou de populisme, de vulgarisation ou de médiation? Comment la circulation et la diffusion des points de vue et des opinions s'effectuent-elles ? Comment peut-on cartographier l'aura et les réseaux de communication des touristes amateurs ? Quelles sont les motivations des touristes blogueurs? Certains blogs prennent des formes qui ne sont pas sans rappeler celles des carnets de voyage (Argod, 20 I0), photos ou croquis à l'appui. Ce supplément d'informations enrichit-il le voyage et l'expérience touristique ou bien renforce-t-il la dimension "parcours imposé" du touriste ? Laisser une trace au monde, prouver son existence, les motivations des touristes sont beaucoup plus profondes que ne le laisserait supposer l'apparence anodine du secteur. Comment réagissent les premiers touristes "accompagnés" dans leur mobilité par des outils mobiles gélocalisés qui leur fournissent quand il le faut et où il le faut l'information désirée?

On le voit, l'invention de la communication touristique est un long chemin, d'autant plus long qu'il évolue avec la technologie. Son but est de comprendre le comment et le pourquoi du tourisme, les mobiles des touristes, les modes de construction de leurs représentations... Il est d'analyser les modes de communication spécifiques, d'étudier les échanges qui se mettent en place, les conséquences pour tous à longue durée des migrations touristiques, la place de la technique dans la relation humaine, etc. Une telle invention ne peut que favoriser le développement d'un tourisme bien géré et accepté par les populations émettrices et réceptrices. Elle ne peut que contribuer à anticiper les problèmes susceptibles de naître à partir de l'échange social qui est la nature même du tourisme et qui fait sa richesse.

\section{RÉFÉRENCES BIBLIOGRAPHIQUES}

Rachid AMIROU, Imaginaire touristique et sociabilités du voyage, Puf, 1995.

Pedro ANDRADE, "Tourism imaginaries and web 3.0: Geneologic methods in the analysis of tourist interaction with urban public art", communication lors de la conférence "Imaginaires touristiques" à Berkeley, Californie, février 2011.

Pascale ARGoD, "Le carnet de voyage audiovisuel ou cinématographique. Genre intermédial, quête et diffusion du voyage 'authentique"', Téoros, 30-I, 201 I.

$\overline{\text { Rodolfo BAGGIO }}$ et Jane KLOBAS,

Quantitative Methods in Tourism. A Handbook, Channel view publications, $201 \mathrm{l}$.

Roland BARTHES, Mythologies, Seuil, 1957.

Jean BAUDRILLARD, Simulacres et simulation,

Galilée, 1991.

$\overline{\text { Françoise BERNARD et Robert-Vincent }}$

JOULE, "Le pluralisme méthodologique en sciences de l'information et de la communication à l'épreuve de la 'communication engageante"', Questions de communication, $n^{\circ} 7,2005$.

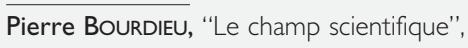
Actes de la recherche en sciences sociales, vol. 2, 1976

Marc BOYER, L'Invention du tourisme,

Gallimard, 1996.

Marc BOYER, Histoire du tourisme de masse, Puf, 1999.

Marc BOYer et Philippe VIALLON,

La Communication touristique, coll. "Que sais-je ?", Puf, 1994.

Rinaldo BrAU, “Demand-driven sustainable tourism? A choice modelling analysis", Tourism Economics, Vol. 14, N 4, 2008. 


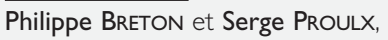

L'Explosion de la communication à l'aube du XXe siècle, La Découverte, 1993.

Sylvie BRUNEL, La Planète disneylandisée.

Chroniques d'un tour du monde, Éd. Sciences

humaines 2006.

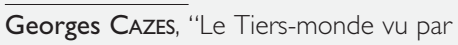
les publicités touristiques : une image mystifiante", Les Cahiers du tourisme, 1976.

Christophe ClivaZ et Stéphane NAHRATH,

"Le retour de la question foncière dans

l'aménagement des stations touristiques

alpines en Suisse", Revue de géographie

alpine/Journal of Alpine, 98-2, 2010 [en ligne:

http://rga.revues.org//220].

Erik COHEN, "Rethinking the sociology of tourism", Annals of Tourism Research, Vol. 6, $n^{\circ} 1,1979$.

Saskia Cousin, L'Identité au miroir du tourisme. Usages et enjeux des politiques de tourisme culturel, thèse de doctorat en anthropologie sociale et ethnologie, 2003 [en ligne : http://tel.archives-ouvertes.fr/tel00266547].

Éric DaCHeuX, Les Sciences de l'information et de la communication, CNRS éditions,

2009, pp. 9-36.

Joffre DUMAZEDIER, Vers une civilisation du loisir?, Seuil, 1962.

Hans-Magnus ENZENBERGER, Culture ou mise en condition?, Belles Lettres, 1965.

Maria Gravari-Barbas et Nelson Graburn,

"Imaginaires touristiques", Via@, nI, 2012

[en ligne :

http://www.viatourismreview.net/PDF/Edito

riall.pdf].

Jean-Michel HOERnER, Traité de tourismologie. Pour une nouvelle science touristique,
Presses universitaires de Perpignan, 2002.

Roman JAKOBSON, Essais de linguistique

générale, Éditions de Minuit, 1963.

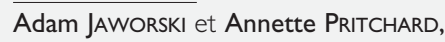

Discourse, communication, and tourism,

Channel view publications, 2005.

Harold LASSWELL, Propaganda technique in the world war, MIT Press, 1927.

Paul Lazarsfeld et Elihu Katz, Personal Influence, the Part Played by People in the Flow of Mass Communications, The Free

Press, 1955.

Sonia LIVINGSTONE, "Audience research at the crossroads The'implied audience'in media and cultural theory", European journal of cultural studies, vol. I, n 2, 1998.

Kurt LUGER, "Kommunikation im

Tourismus. Projektskizze für eine kommunikationswissenschaftliche

Tourismusforschung", Aurora, Magazin für Kultur, Wissen und Gesellschaft, 2000 [en ligne : http://www.aurora-magazin.at/gesellschaft/luger_frm.htm].

Armand MATTELART, L'Invention de la communication, La Découverte, 1994.

ÉQUIPE MIT, Tourismes I. Lieux communs, Belin, 2002.

ÉQUIPE MIT, Tourismes 2. Moments de lieux, Belin, 2005.

ÉQUIPE MIT, Tourismes 3. La révolution durable, Belin, 201।.

Jean-Claude Morand et Brice Mollard, Tourisme 2.0. Préparer son voyage, préparer son offre de tourisme, M2I Éditions, 2008.

Pierre Musso, Critique des réseaux, Puf, 2003.

Stéphane NAHRATH, Jean-David Gerber, Peter Knoepfel et Christian Bréthaut, 
"Gestion des ressources communes en Suisse : le rôle des institutions de gestion communautaire dans les politiques environnementales et d'aménagement du territoire", Nature, sciences et société, vol. $20, n^{\circ}$ I, 2012.

Ronald NYKIEL, Handbook of Marketing Research Methodologies for Hospitality and Tourism, Rootledge, 2007.

OMT, "Le tourisme international en voie d'atteindre le milliard de touristes à la fin de 2012", communiqué de presse, 2012 [en ligne : http://media.unwto.org/fr/pressrelease/2012-09-13/le-tourisme-international-en-voie-d-atteindre-le-milliard-de-touristes-la-f].

Tijana RAKIC et Donna Chambers (dir.), An Introduction to Visual Research Methods in

Tourism, Routledge, 2012.

Greg Richards et Wil Munsters, Cultural

Tourism Research Methods, Cabi, 2010.

C. E. SHANNON, "A mathematical theory of communication", Bell System Technical Journal, juillet 1948 [disponible sur http://circuit.ucsd.edu/ yhk/ece287awin08/shannon 1948.pdf].

\section{Claude-Henri DE SAINT-SIMON,}

L'Organisateur, éditions Dentu, 1819.

Mathis Stock, Le Tourisme. Acteurs lieux et enjeux, Belin, 2003.

Stratégies, "Internet fait voyager les Français", 2009 [en ligne : http://www.strategies.fr/blogs-opinions/ideestribunes/ I 293 I 7W/internet-fait-voyagerles-francais.html].

Laurent TIssot, “Le tourisme : de l'utopie réalisée au cauchemar généralisé ?",

Entreprises et histoire, vol. 2, n 47, 2007.

Gérard TOCQUER et Michel ZINS, Marketing du tourisme, Gaëtan Morin, 1997.

Jean-Didier URBAIN, L'ldiot du voyage : histoires de touristes, Plon, 1991.

Jean-Didier URBAIN, Sur la plage. Mœurs et coutumes balnéaires, Payot, 1994.

\section{Mazaffer UYSAL et Claudia JUROWSKI,}

"Testing the push and pull factors", Annals of Tourism Research, vol. 21, n 4, 1994.

Thorstein VEBLEN, The Theory of the Leasure Class: an Economic Study of Institutions,

Macmillan, 1899.

Philippe VIALLON, "Immigration et tourisme : prolégomènes à une approche interculturelle de ces phénomènes", dans Larbi CHOUIKHA, Vincent MEYER et Wahid GDOURA (dir.), Interagir et transmettre, informer et communiquer : quelles valeurs, quelle valorisation ?, Tunis, ISD, IPSI, SFSIC, 2008.

\section{Philippe Viallon et Yeny Serrano,}

"Appropriation du web 2.0 dans le secteur du tourisme : 'empowerment' des internautes?", communication lors du séminaire du Lisec, Gérardmer, juin 2013.

\section{Philippe VIALlon et Sandrine HenNeke-}

LANGE, "Sites internet touristiques : invitation au voyage ou adhésion à un mythe?", Actes du colloque "Recherches en communication, Images, récits et nouveaux médias : de la transmission à la coconstruction de la croyance", à paraître (20|3).

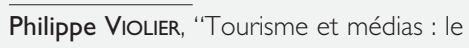
regard d'un géographe", Le Temps des médias, vol. I, n 8, 2007.

Paul WatzlaWick (dir.), L'Invention de la réalité. Contributions au constructivisme, Seuil, 1988.

Norbert WIENER, Cybemétique et société,

Union générale d'éditions, 1952. 